\title{
Enseñanza-aprendizaje de la electrónica digital basa- do en niveles de complejidad de programación
}

\author{
Teaching-learning in digital electronics based on complexity levels \\ in programming
}

\author{
Luis Alexánder Jiménez Hernández ${ }^{1}$ \\ Ignacio Laiton Poveda ${ }^{2}$
}

\section{Resumen}

En este artículo se presentan los aspectos principales del proyecto "Etapa de análisis de aprendizajes en estudiantes empleando el prototipo para procesos de enseñanza-aprendizaje de la electrónica digital con dispositivos programables en la especialidad técnica mecatrónica", financiado por la Escuela Tecnológica Instituto Técnico Central (Conv. 04, 2016). Abarca la formulación y aplicación de una interacción didáctica, con el propósito de evaluar los avances en el aprendizaje de las temáticas relacionadas con la Electrónica Digital. Como resultados de investigación, se espera obtener información relevante acerca de los aprendizajes de los estudiantes de Electrónica Digital basada en la metodología didáctica formulada para el desarrollo del curso y apoyada en el uso del prototipo diseñado en la etapa previa a este proyecto.

\section{Palabras clave: didáctica, tecnologías para la educación, dispositivos propios, electrónica Digital}

\section{Abstract}

The following article describes the main aspects in the project entitled "Learning Analysis Stage in students using a prototype for teaching-learning processes in digital electronics with programmable devices in the Technical Specialty of Mechatronics ", this project was financed by the Technological School "Instituto Tecnico Central" (Call 04, 2016). The project includes the manner and the implementation of a teaching approach interaction, for the purpose of evaluating the learning advances in the subjects related to Digital Electronics. As a result of this research, important information about different learning styles in Digital Electronics students are expected to be obtained based on the methodological didactic drew up during the development of the course, and supported in the use of the prototype designed in the previous stage to this project.

\section{Keywords: Didactics, Technology for education, Own devices, Digital Electronics}

Citación del artículo:

Jiménez, L. A., y Laiton, I. (2018). Enseñanza-aprendizaje de la electrónica digital, basado en niveles de complejidad de programación. Letras Conciencia Tecnológica, 18, 01-10.

1 Doctor en Educación, magíster en Educación, Ingeniero Electrónico. Docente de planta en la Escuela Tecnológica Instituto Técnico Central ETITC. Correo electrónico: alexanderjimenez77@yahoo.com

2 Doctor en Educación, Magíster en Docencia, Licenciado en Física. Docente de la ETITC y director del grupo de investigación en Pedagogía de las Ciencias Básicas - GIPEC. Correo electrónico: ilaiton@itc.edu.co 


\section{Introducción}

A partir de la formulación de la especialidad técnica denominada Mecatrónica en el Instituto de Bachillerato Técnico Industrial - IBTI, se plantearon varias actualizaciones que abarcaron aspectos metodológicos y curriculares, orientadas a la conformación de un plan de estudios que ofreciera mayores beneficios a los estudiantes en términos de aprovechamiento y significancia de sus experiencias de aprendizaje (Osorio y Rojas, 2010). Como resultado, se formuló una planeación académica compatible con los contenidos temáticos y los tiempos de dedicación de los estudiantes, en relación con varias asignaturas disciplinares de la estructura curricular del primer ciclo profesional de la Facultad de Mecatrónica, programa académico ofrecido por la Escuela Tecnológica Instituto Técnico Central - ETITC (Jiménez, 2013).

Dadas las condiciones mencionadas, a los estudiantes se les han reconocido sus avances en estudios de formación técnica profesional mediante normativa institucional (Acuerdo 14, 2013; Propuesta de Actualización, 2016c), confiriendo la homologación de varias asignaturas de la Especialidad Técnica Mecatrónica ETM, particularmente para el ingreso al programa Técnico Profesional en Electrónica Industrial TPEI, correspondiente al primer ciclo propedéutico del programa de Mecatrónica de la ETITC. Las homologaciones mencionadas se plantearon a partir de los correspondientes estudios y procesos de actualización curricular (ETITC, 2016a). En los cuatro años transcurridos desde la formulación y puesta en marcha del proceso de articulación y continuidad formativa entre la ETM y el programa TPEI, figura la homologación de la asignatura Electrónica Digital como componente fundamental de formación en la línea disciplinar de la electrónica básica.

De otra parte, considerando la preparación disciplinar del estudiante en formación técnica en el campo de la Mecatrónica, se reconoce que los temas relacionados con la Electrónica Digital en- vuelven aspectos primordiales sobre el diseño y la operación de dispositivos electrónicos para el control digital que conforman la mayoría de sistemas mecatrónicos (Bolton, 2001). Además muchas de las aplicaciones digitales de control abarcan o se basan en el uso de sistemas programables embebidos que se encargarán del procesamiento de la información de manera autónoma, es decir, que realizarán sus funciones de control sin asistencia de un computador. De igual manera, con la programación textual los estudiantes aprenden el uso de un lenguaje formal estructurado, propiciando la comprensión para la planeación, seguimiento y ejecución de órdenes algorítmicas, principios que son empleados en el campo de la robótica industrial (Barrientos, Peñin, Balaguer y Aracil, 1997).

Cabe agregar que, dada la trayectoria de la ETITC en el ámbito de formación para el sector industrial, el diseño curricular en el área técnica del bachillerato corresponde a una modalidad educativa basada en el desarrollo de competencias. Este enfoque es reciente en el campo educativo y, por lo tanto, las pautas para su asimilación y aplicación en los escenarios de enseñanza tradicional no han sido aún suficientes para su puesta en práctica, más cuando ha sido objeto de críticas por sus orígenes instrumentalistas, respecto al ámbito de donde proviene, caracterizado por reglas económicas de oferta y demanda (Díaz, 2011). No obstante, se pueden identificar dos tendencias que guían actualmente la formación de carácter técnico: la primera corresponde a la preparación para un desempeño laboral, basada en la aplicación eficiente de conocimientos disciplinares; la segunda basada en la relación entre lo actitudinal y lo comportamental en los ámbitos personal y social del futuro trabajador (Roth, 2017).

\section{Metodología}

Algunos resultados de investigación previos permitieron identificar unas necesidades en cuanto al mejoramiento de los aprendizajes (ETITC, 2014), a las prácticas educativas desde lo instruccional y lo 
curricular (Mooij, 2009) y la reacción emocional de los estudiantes (Bower y Forgas, 2003). Además, se establecieron estrategias metodológicas como disminuir el tiempo de acceso a las tecnologías de diseño electrónico digital (Jiménez, 2014a), y reducir la dependencia de disponibilidad de salas de cómputo con software especializado (Draghiciu y Pepeneata, 2014). También se buscó responder a las necesidades analizadas desde el inicio del proceso de articulación entre el bachillerato técnico con la educación superior acerca del nivel de desarrollo de los estudiantes (Jiménez, 2013).

A partir de las estrategias mencionadas, se consideró pertinente que los estudiantes participaran en los ejercicios prácticos obteniendo resultados satisfactorios, de manera que acumulen experiencias significativas que recompensen su dedicación y esfuerzo. Tal planteamiento condujo a una propuesta didáctica de aprendizajes por niveles, como se ilustra en la figura 1. De igual manera, puede entenderse que el docente tiene gran responsabilidad en la generación de escenarios de trabajo académico en condiciones pedagógicas favorables, y que puede apoyarse en tecnologías didácticas que faciliten la creación de ambientes de aprendizajes adecuados para sus estudiantes (Roth, 2017).

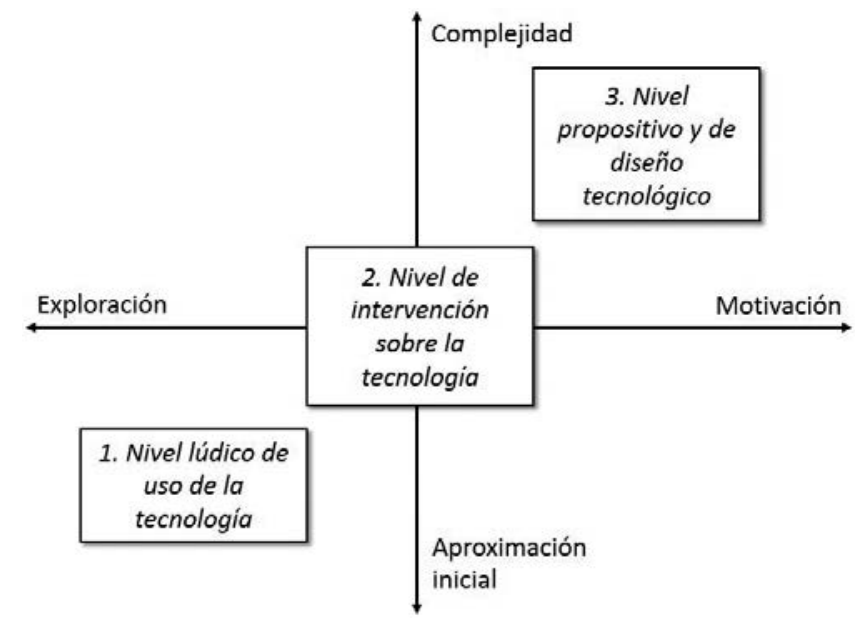

Figura 1. Modelo metodológico por niveles de aprendizaje planteado en el proyecto para los procesos de enseñanza-aprendizaje en el curso de la electrónica digital. Fuente autor
Acorde al planteamiento metodológico en la etapa inicial de diseño (Jiménez, 2015b), en el proyecto se formuló un marco de investigación cualitativa, buscando establecer los elementos que aportan al aprendizaje de los estudiantes y faciliten la comprensión y usos de las tecnologías digitales programables. En la primera fase, se desarrolló un análisis descriptivo sobre las actividades del curso de Electrónica Digital, buscando establecer las posibilidades de uso práctico del prototipo en el laboratorio con los estudiantes con base en experiencias previas desde la perspectiva del docente. Para la segunda fase se realizó el proceso de interacción con los estudiantes basado en la propuesta didáctica que involucra el uso del prototipo y en la que se realiza el seguimiento a los aprendizajes a través de instrumentos.

Las características demográficas del grupo de interacción son: 36 estudiantes (curso de Electrónica Digital de la Especialidad Técnica Mecatrónica, grado $9 .^{\circ}$ ) distribuidos en dos grupos, con edades cercanas a los 15 años y de género mixto. Para la recolección de la información, se ha planteado emplear técnicas de evaluación con instrumentos aplicados a los estudiantes durante el periodo de interacción, con el propósito de obtener datos en torno a categorías de análisis que permitan analizar cualidades particulares del uso del prototipo como herramienta de aprendizajes, y profundizar posteriormente empleando técnica de entrevistas semiestructuradas, con el fin de complementar aspectos relevantes como concepciones y representaciones de los estudiantes (Jiménez, 2015a).

Entre los resultados de la presente investigación se encuentran, en primer lugar, el diseño e implementación de una metodología basada en la resolución de problemas como eje formativo de la ETM, de acuerdo con un análisis descriptivo precedente sobre la modalidad de procesos de enseñanza-aprendizaje (Jiménez, 2014b). También se espera beneficiar a los estudiantes de la Especialidad Técnica Mecatrónica, mejorando aspectos relacionados con el aprendizaje de las tecnologías 
digitales programables útiles para diseñar e implementar sistemas mecatrónicos (Bolton, 2001). En segundo lugar, los docentes que imparten las asignaturas sobre el tema de tecnologías digitales podrán hacer uso de la herramienta didáctica (ver figura 2) y la propuesta metodológica como fundamento o complemento didáctico de las actividades prácticas en el laboratorio en cursos relacionados con las tecnologías digitales (cursos de computación y de control digital) en los niveles de formación técnico, tecnológico y profesional.

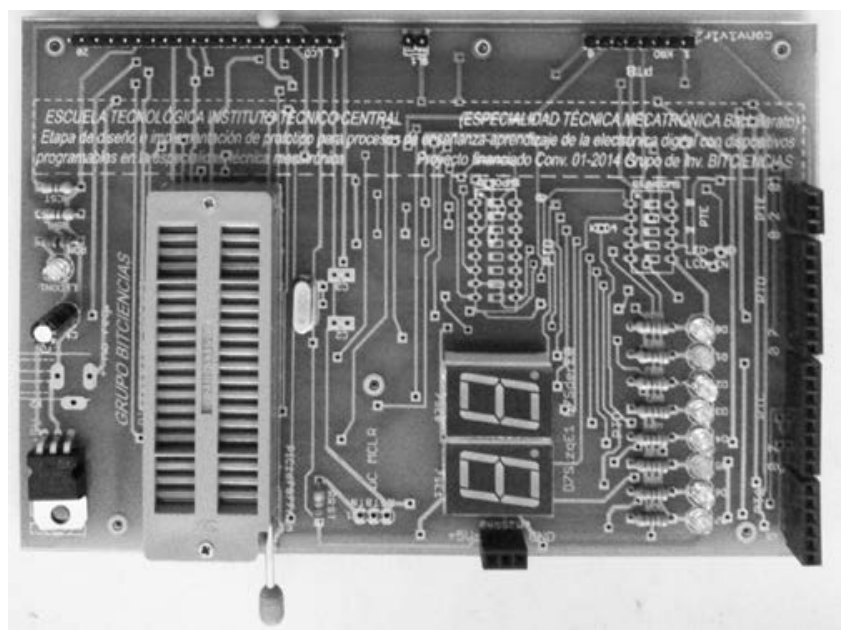

Figura 2. Montaje del hardware de la herramienta didáctica desarrollada para el proyecto. El prototipo puede ser empleado en los tres niveles de aprendizaje planteados en la propuesta metodológica.

Fuente: autores

\section{Conclusiones}

Finalmente, en la primera fase del proyecto se determinaron los siguientes aspectos sobre el uso tecnológico: se logró el diseño del prototipo de acuerdo a los aspectos didácticos planteados como herramienta tecnológica para un curso de Electrónica Digital de la Especialidad Técnica Mecatrónica, bajo los fundamentos de aprendizajes por niveles de complejidad orientados a mejorar el acceso y la intervención de los estudiantes en un diseño electrónico flexible. Además, los estudiantes cuentan con la posibilidad de modificar el diseño inicial del prototipo para ser adaptado de acuerdo con los requisitos del nuevo diseño, lo que permitiría ser empleado como estructura base de hardware y software para otras asignaturas orientadas por la metodología de proyectos empleando tecnologías propias orientadas al desarrollo tecnológico electrónico, o también para realizar proyectos de grado en los distintos niveles educativos (bachillerato técnico industrial, ciclo técnico profesional, tecnológico y profesional de ingeniería).

De acuerdo con lo anterior, el uso tecnológico de la herramienta en el espacio didáctico de los procesos de enseñanza-aprendizaje de la electrónica digital compete a los niveles formativos de secundaria técnica y de educación superior. A partir de los desarrollos logrados mediante esta modalidad de uso tecnológico se puede conformar un escenario para el futuro diseño de recursos didácticos, para la profundización a distintos niveles en otras áreas de estudio de la electrónica, más allá de las tecnologías disponibles en el mercado industrial y educativo. Por otra parte, mediante la metodología de trabajo con tecnologías propias, los estudiantes pueden apropiarse más fácilmente de temas orientados al diseño industrial, como la caracterización de diferentes componentes, uso de dispositivos y materiales nuevos, técnicas de diseño, simulación de circuitos electrónicos y conocimientos en formulación y evaluación de proyectos.

\section{Referencias bibliográficas}

Barrientos, A., Peñin, L. F., Balaguer, C., y Aracil, R. (1997). Fundamentos de robótica. México: Editorial McGraw Hill.

Bolton, W. (2001). Mecatrónica: sistemas de control electrónico en ingeniería mecánica y eléctrica (2a edición). México: Alfaomega.

Bower, G. H., y Forgas, J. P. (2003). Afecto, memoria y cognición social. En J. Aldekoa (Trad.), Cognición y Emoción (pp. 82-169). Bilbao: Desclée de Brower. 
Díaz-Barriga, A. (2011). Competencias en educación. Corrientes de pensamiento e implicaciones para el currículo y el trabajo en el aula. Revista Iberoamericana de Educación Superior, 2(5), 3-24. Recuperado de http:// ries.universia.net/index.php/ries/article/ download/126/pdf_1

Draghiciu, N., y Pepeneata, A. (2014). Educational purpose cardiopulmonary resuscitation simulator [Simulador de resucitación cardiopulmonar con propósitos educativos]. Journal of Electrical and Electronics Engineering, 7(1), 39-42. Recuperado de https://www. ebscohost.com/

Escuela Tecnológica Instituto Técnico Central. (21 de enero de 2016a). Acta de reunión n.o 0304. Programa de Ingeniería Mecatrónica. Documento de trabajo.

Escuela Tecnológica Instituto Técnico Central. (21 de enero de 2016b). Acta de reunión n. $\mathrm{o}$ 0721. Programa de Ingeniería Mecatrónica. Documento de trabajo.

Escuela Tecnológica Instituto Técnico Central. (10 de diciembre de 2013). Acuerdo 14 por el cual se autoriza homologar asignaturas de los programas de educación superior a los bachilleres del Instituto de Bachillerato Técnico Industrial - ETITC. Bogotá: ETITC.

Escuela Tecnológica Instituto Técnico Central. (2016c). Propuesta de actualización de homologaciones de asignaturas entre Especialidad Técnica Mecatrónica de Bachillerato y Programa Técnico Profesional en Electrónica Industrial. Equipo de trabajo Especialidad Técnica Mecatrónica. Documento de trabajo.

Escuela Tecnológica Instituto Técnico Central. (2014). Propuesta del proyecto "Etapa de diseño e implementación de prototipo para procesos de enseñanza-aprendizaje de la electrónica digital con dispositivos programables en la especialidad técnica mecatrónica". Vicerrectoría de Investigación. Documento de trabajo.

Escuela Tecnológica Instituto Técnico Central. (14 de febrero de 2017). Proyecto Curricular Especialidad Técnica Mecatrónica año 2017, aprobado en Consejo Académico del IBTI. Equipo de trabajo Especialidad Técnica Mecatrónica. Documento de trabajo.

Jiménez, L. A. (septiembre, 2013). Articulación entre programas de especialidades técnicas de bachillerato con la educación superior en el área de ingeniería: pasos iniciales para la formación temprana de ingenieros en Colombia. Cartagena de Indias: paper presentado en el World Engineering Education Forum WEEF 2013, Innovación en Investigación y Educación en Ingeniería: factores claves para la competitividad global.

Jiménez, L. A. (2014a). Acceso a la electrónica digital mediante herramientas didácticas basadas en el desarrollo de dispositivos propios. Manizales: ponencia presentada en la IX Conferencia Latinoamericana de Objetos y Tecnologías de Aprendizaje.

Jiménez, L. A. (2014b). Procesos educativos de le especialidad técnica mecatrónica: una asociación entre el aprendizaje significativo y la resolución de problemas. En N. Murcia. (Comp.), Sistematización de Experiencias Educativas en la Escuela Lasallista, (cap. 14). Bogotá: Editorial Congregación de los Hermanos de las Escuelas Cristianas.

Jiménez, L. A. (2015a). Desarrollo metacognitivo enfocado en procesos de monitoreo $y$ control en estudiantes de secundaria técnica, empleando el modelo de resolución de problemas en una perspectiva de 
investigación. (Tesis doctoral sin publicar). Bogotá: Universidad Santo Tomás.

Jiménez, L. A. (2015b). Proyecto etapa de diseño e implementación de prototipo para procesos de enseñanza aprendizaje. Letras Conciencia Tecnológica, 13, 60-67.

Mooij, T. (2009). Education and ICT-based self-regulation in learning: Theory, design and implementation [Educación y autorregulación en el aprendizaje basado en TIC: teoría, diseño e implementación].
Education and Information Technologies 14, 3-27. Doi: 10.1007/s10639-008-9066-8

Osorio, E., y Rojas, A. (2010). Currículo propuesto para el Bachillerato Técnico Industrial en Mecatrónica. Letras Conciencia Tecnológica, 8, 27-37.

Roth-Berlin, S. (12 de octubre de 2017). Sistema educativo de modalidad dual alemán. Conferencia presentada en la Transferencia Internacional de Conocimiento ETITC-FESTO, Vocational School Philipp-Matthäus-Hahn, Balingen. 